\title{
INTEGRANDO ACTIVIDADES DE GESTIÓN DE LA INFORMACIÓN EN EDUCACIÓN SUPERIOR. UNA EXPERIENCIA EN INFORMÁTICA EDUCATIVA
}

\author{
Susana Molina Martín \\ Mª Teresa Iglesias García \\ Carmen Diego Pérez \\ Universidad de Oviedo
}

\begin{abstract}
RESUMEN: Una importante misión de las instituciones de educación superior es formar personas capaces de aprender a lo largo de la vida, a cuyo logro contribuye la competencia gestión de la información. El entorno actual, en el que proliferan nuevos recursos de información, hace que los individuos necesiten nuevas habilidades relacionadas con la localización, evaluación y uso eficaz de la información. Por ello, presentamos en este trabajo una experiencia cuyo principal objetivo ha sido desarrollar diversas habilidades relacionadas con la gestión de la información mediante su integración en las actividades de la asignatura Informática Educativa de la Licenciatura de Pedagogía de la Universidad de Oviedo. Los resultados muestran que el nivel de los estudiantes en algunas de estas habilidades, como el diseño e implementación de una estrategia de búsqueda, la evaluación crítica de la información obtenida o la elaboración de conclusiones a partir de su lectura, es insuficiente, por lo que sería recomendable que los futuros planes de estudio de la Universidad integren esta competencia en los programas de las materias.
\end{abstract}

ABSTRACT: Developing lifelong learners is central to the mission of higher education institutions. Information literacy is a key component of, and contributor to, lifelong learning. Because of rapid technological change and proliferating information resources, individuals required new skills to locate, evaluate and use information effectively. In this context, the main aim of this experience was students learn different information literacy skills. For that, incorporate information literacy in the activities of the subject Education Computer Science was needed. Results showed that students' level in skill as selects the most appropriate information retrieval system, constructs and implements effectively designed search strategies, critically evaluates the procured information and draws conclusion based on upon information 
gathered, were inadequate, so universities should integrate information literacy as much possible in subjects' programs.

PALABRAS CLAVE: Alfabetización en información, educación permanente, tecnologías de la información y de la comunicación, habilidades de información, educación superior, innovación.

KEYWORDS: Information literacy, lifelong learning, information and communication technologies, information skills, higher education, innovation.

\section{INTRODUCCIÓN}

En la denominada sociedad de la información y del conocimiento, las personas se encuentran en situación de tener que tomar diversas decisiones que afectan a su vida personal, académica y profesional, para las que requieren estar informadas. La proliferación de nuevos recursos, la aparición de Internet y otras posibilidades electrónicas y digitales han propiciado tanto el incremento de la información disponible como modificaciones en la forma de crearla, almacenarla y distribuirla. Estos cambios no han ido acompañados de suficientes mecanismos de control de calidad y validez de la información, ni de elementos para asegurar una mayor precisión en su representación y organización. Existe el riesgo de que la información valiosa se pierda entre otra que resulta obsoleta, redundante, imprecisa o mal estructurada, lo que se convierte en un verdadero problema si se carece de formación para la gestión de la información. De ahí que haya cobrado fuerza la idea de que para vivir en la sociedad actual no es suficiente, aunque sí necesario, el acceso libre y gratuito a la información sino que además se requiere de una formación dirigida a alcanzar la autonomía en la identificación, localización, selección, valoración, contraste, tratamiento y utilización eficaz y ética de la información proveniente de distintas fuentes para responder a un problema planteado, tomar decisiones o construir conocimiento. Las habilidades descritas forman parte de la denominada Alfabetización Informacional -ALFIN- (American Library Association,1989), considerada desde instancias internacionales un derecho humano básico y crucial para el desarrollo de regiones y naciones en una economía basada en el conocimiento, como puede leerse en la Declaración de Praga (United Nations Educational, Scientific and Cultural Organization \& National Forum on Information Literacy, 2003) y en la Declaración de Alejandría (United Nations Educational, Scientific and Cultural Organization \& National Forum on Information Literacy, 2005).

Las universidades, conscientes tanto de la importancia de adquirir un nivel adecuado en estas habilidades para que el individuo pueda seguir aprendiendo a lo largo de la vida e integrarse plenamente en el entorno social como de las carencias formativas al respecto, están tratando de fomentar su desarrollo. Para un aprendizaje exitoso, la labor formativa en ALFIN Ilevada a cabo por las bibliotecas debe complementarse con la intervención del profesorado. Pinto, Sales y Martínez-Osorio (2009) en su trabajo dirigido a realizar un diagnóstico de la relación que tienen con ALFIN los profesionales de las bibliotecas universitarias en España, concluyen con un breve análisis DAFO, señalando entre las fortalezas "el reconocimiento que el colectivo académico otorga a la importancia de las habilidades informacionales como parte innegable del currículum formativo de su alumnado" (78) y entre las 
oportunidades en el marco de la convergencia europea "integrar la formación ALFIN de forma sólida y transversal en el currículum formativo de grado y postgrado" (78).

Algunos autores, como Webber y Johnston (2006), consideran que el desarrollo adecuado en esta competencia se conseguirá mediante lo que han denominado una Universidad alfabetizada en información, lo que supone integrarla tanto en el currículo como en todos los programas y servicios ofertados. En esta línea, han comenzado a darse pasos a favor de la inclusión de esta competencia básica en los planes de estudio tanto a nivel nacional como internacional (Bernhard, 2002). En España, en numerosos documentos surgidos a raíz del proceso de convergencia europea ha sido reconocida como una competencia básica y necesaria para vivir y trabajar en esta nueva era digital, como es el caso de los libros blancos del título de grado en Pedagogía (Agencia Nacional de Evaluación, Calidad y Acreditación, 2005: 142) y en los requisitos para la verificación de los títulos universitarios oficiales de Maestro en Educación Infantil y Primaria (Ministerio de Educación y Ciencia, 2007c; 2007d). En cuanto a las nuevas titulaciones que se están elaborando, incorporan el desarroIlo de esta competencia de dos formas complementarias y enriquecedoras. En primer lugar, mediante la creación de una asignatura específica que los estudiantes tienen que cursar en los programas de grado e incluso en los de postgrado, dirigida fundamentalmente al desarrollo de los procedimientos implicados en la búsqueda y uso de la información específica de la titulación. Tal es el caso de la Universidad de Oviedo, donde ya se introdujo en el plan de estudios de la titulación de Licenciado en Pedagogía (Universidad de Oviedo, 2000) la asignatura obligatoria Sistemas de Información y Documentación sobre Educación, cuyo contenido permanecerá en el titulo de Grado en Pedagogía del futuro plan de estudios bajo la denominación Gestión de la Información en Educación. En segundo lugar, mediante la integración de esta competencia en la docencia universitaria de todas las asignaturas, en el modelo de enseñanza adoptado por el profesorado, pues su desarrollo requiere y depende de que los estudiantes pongan en práctica las habilidades implicadas.

La experiencia que aquí se presenta se sitúa en el último contexto señalado, al tratar de integrar el desarrollo de algunas de las habilidades implicadas en la gestión de la información en el proceso de enseñanza-aprendizaje de la asignatura Informática Educativa de la titulación de Licenciado en Pedagogía. Partimos del hecho de que aunque en la Facultad de Ciencias de la Educación de la Universidad de Oviedo hay una asignatura completa, anual, obligatoria y que cuenta con noventa y cinco horas lectivas presenciales, dirigida específicamente al desarrollo de esta competencia, ésta no resulta suficiente para su logro, pues al tener el carácter de herramienta procedimental necesita ser puesta en práctica para su generalización (Molina, Iglesias y Diego, 2008). Finalmente, cabe decir que esta innovación docente forma parte de un proyecto más amplio que ha sido financiado por el Vicerrectorado de Calidad, Planificación e Innovación, de la Universidad de Oviedo, España (Referencia: PB-08-022).

\section{DESCRIPCIÓN DE LA EXPERIENCIA}

La asignatura Informática Educativa, una de las tres en la que se desarrolló esta experiencia, se imparte en el primer cuatrimestre y consta de cuarenta y cinco horas 
lectivas presenciales. Su carácter optativo favorece que se matricule en ella alumnado procedente de distintos cursos de la propia titulación y de otros estudios universitarios, por tanto, con conocimientos previos muy diversos. Únicamente los estudiantes que inician Pedagogía en primero han cursado la asignatura obligatoria Sistemas de Información y Documentación sobre Educación, que los inicia en la gestión de la información. De los treinta y cuatro estudiantes matriculados en la asignatura de Informática Educativa en el curso 2008-2009, únicamente veintiocho participaron de forma continuada en las actividades de la clase.

En el diseño de la mencionada asignatura optativa se incluyeron objetivos específicos propios y otros relacionados con la gestión de la información. Respecto a ésta última, se pretendió mejorar las habilidades de los estudiantes para:

- acceder a la información requerida de manera eficaz y eficiente, mediante el uso de las Tecnologías de la Información y la Comunicación;

- evaluar la información obtenida y sus fuentes;

- usar eficazmente de la información obtenida para la realización de la actividad;

- usar éticamente las fuentes de información utilizadas.

Para ello, se diseñó un taller presencial, con una duración de nueve horas, dirigido a la elaboración de una «Wiki», en el que se tomaron en consideración algunos referentes relacionados con ALFIN. En primer lugar, las normas de alfabetización en información, destinadas tanto a orientar el desarrollo de los programas de formación como a servir de referentes para evaluar su posesión o nivel de dominio, desarrolladas por organizaciones de tres países: la «Association of College and Research Libraries»-ACRL-y «American Library Association» (2000) en Estados Unidos; el «Australian and New Zealand Institute for Information Literacy» (2003) y la «Council of Australian University Librarians»-CAUL- (2002, 2004) en Australia y la «British Society of College, National and University Libraries» (2003) en Reino Unido. En segundo lugar, se tuvieron en cuenta algunos modelos diseñados desde distintos enfoques para la solución de problemas de información, por ejemplo, Estudios de Información kinder a grado 12 formulado por Osla, Búsqueda de Información cuyo autor es Kuhithau, Competencias para el Manejo de la Información -CMIcreado por Irving, Proceso de Investigación de Stripling y Pitts y el Modelo Big6 desarrollado por Mike Eisenberg y Bob Berkowitz (recogidos por Calderon y De Jorge, 2006).

A partir de la revisión de las mencionadas fuentes se diseñó el taller, que se organizó en tres fases de tres horas presenciales cada una. En la Fase I, los estudiantes localizaron y seleccionaron información proveniente de diversas fuentes. En la Fase $I I$, utilizaron la información para resolver la actividad planteada, teniendo en cuenta los aspectos éticos de la misma. En la Fase III, crearon una «Wiki» en grupo y participaron en las «Wikis» de sus compañeros, con la intención de que la colaboración permitiese el perfeccionamiento de la tarea realizada.

En la Fase I, los estudiantes formaron grupos de cuatro componentes de distinta procedencia o curso, para seguidamente:

- Identificar aquellas fuentes dónde buscarían la información necesaria para resolver la actividad. Se comenzó realizando una dinámica de grupo mediante 
la técnica "lluvia de ideas", lo que favoreció que salieran a la luz sus conocimientos previos sobre diversos recursos on-line, bases de datos, etc., y facilitó posteriormente que cada grupo decidiera dónde quería buscar la información.

- Planificar la búsqueda de información, identificando las fuentes relevantes y accediendo a la información que contenían, anotando dónde buscaron y lo qué encontraron en la Tabla 1.

- Valorar la adecuación de la información seleccionada para la elaboración de la «Wiki» y recogerla en la Tabla 1.

- Evaluar la calidad de la información recogida y sus fuentes, a partir de pautas como: tipo de formato, actualidad, autoría, identificar el país o cultura de procedencia, tipo de fuentes, etc. Se pidió a cada uno de los grupos que pusieran una cruz en rojo al lado de los documentos recogidos en la Tabla 1 que desechaban de la búsqueda realizada y que explicasen el motivo.

- Replantear la búsqueda de información para subsanar las carencias detectadas, tras informarles acerca de algunas herramientas y estrategias de búsqueda de información, y recoger la nueva información en la Tabla 1.

Tabla 1. Registro de acceso, selección y evaluación de la información.

Nombre y apellidos de los componentes del grupo:

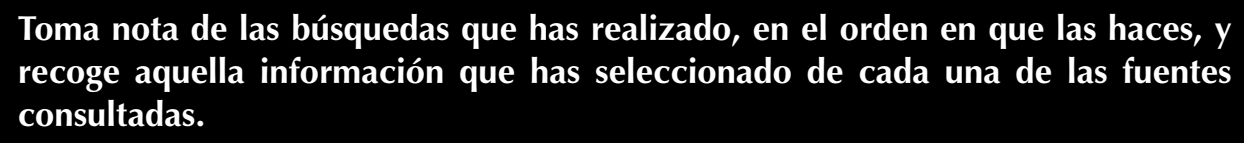

\begin{tabular}{|l|l|l|}
\hline $\begin{array}{l}\text { BÚSQUEDA } \\
\text { REALIZADA (1) }\end{array}$ & \multicolumn{1}{|c|}{$\begin{array}{c}\text { DOCUMENTACIÓN } \\
\text { SELECCIONADA (2) }\end{array}$} & APARTADO (3) \\
\hline & $\begin{array}{l}\text { Identificar el documento: } \\
\text { Evaluación: } \\
\text { Justificación: }\end{array}$ & \\
\hline & $\begin{array}{l}\text { Identificar el documento: } \\
\text { Evaluación: } \\
\text { Justificación: }\end{array}$ & \\
\hline
\end{tabular}

(1) Indicar la fuente (nombre de la base de datos, buscador, etc.)

(2) Recoger aquellos datos que permitan volver a encontrar o identificar al documento y valorar la calidad de la información y sus fuentes.

(3) Indicar la página de la Wiki para el que resulta pertinente la información obtenida. 
En la Fase II, se realizaron tres acciones: 1) Cada estudiante se responsabilizó de la creación del texto de una de las páginas de la «Wiki», entremezclando, estructurando y usando éticamente la información seleccionada. 2) Revisado el texto individual, detectando las carencias, se procedió a explicar al grupo clase la necesidad de usar éticamente la información utilizada, citando las fuentes utilizadas y registrando las referencias bibliográficas. 3) Cada estudiante revisó el texto elaborado y lo completó, incorporando citas y referencias, ordenado las ideas, etc.

En la Fase III, cada grupo de estudiantes creó su «Wiki» a partir de las aportaciones individuales, que fueron revisadas y corregidas por sus miembros. Esta versión de la «Wiki» fue valorada por el profesorado con la intención de hacer sugerencias a cada uno de los grupos para mejorar la calidad de los contenidos, la redacción, la presentación, etc. Cada grupo, tras introducir las modificaciones correspondientes en su «Wiki», procedió a participar en las «Wikis» de otros grupos, de cara a su mejora. Finalmente, cada grupo revisó el estado de su «Wiki» y elaboró una versión definitiva.

La evaluación de esta actividad fue continua durante el desarrollo del taller, recogiéndose información sobre las habilidades manifestadas de los estudiantes para acceder, seleccionar y evaluar la información mediante: 1) Las expresiones orales durante la dinámica grupal, lo que permitió hacerse una idea acerca de los conocimientos previos del alumnado sobre las fuentes y el acceso a la información. 2) El trabajo escrito presentado en la Tabla 1 que había completado cada grupo de estudiantes en dos momentos distintos del desarrollo de la Fase I. También se recogió información sobre las habilidades de los estudiantes en el uso eficaz y ético de la información mediante: 1) El primer documento elaborado por cada uno de los estudiantes para la «Wiki» en la Fase II. 2) La primera versión de la «Wiki» elaborada por cada uno de los grupos en la Fase III. 3) La versión final de la «Wiki».

Para valorar el progreso del alumnado en cada uno de los objetivos en los distintos momentos de desarrollo del taller y reorientar el proceso de enseñanza-aprendizaje, se elaboraron y usaron los criterios, dimensiones y niveles que se especifican en la Tabla 2, elaborados teniendo en cuenta las propuestas de la ACRL (2008), de la CAUL (2002) y de la «Australian Library and Information Association» (2003). El nivel I expresa el mayor nivel de desarrollo de la habilidad, en el nivel II se encontrarían aquellos estudiantes con un menor nivel de destreza y en el nivel III aquellos que no siguen casi ninguna de las pautas pertinentes. 
Tabla 2. Criterios, dimensiones y niveles de logro de los objetivos.

\begin{tabular}{|c|c|c|c|c|}
\hline \multirow{2}{*}{ Objetivos } & \multirow{2}{*}{ Criterios } & \multicolumn{3}{|c|}{ Dimensiones } \\
\hline & & Nivel I & Nivel II & Nivel III \\
\hline $\begin{array}{l}\text { Acceder } \\
\text { eficaz y } \\
\text { eficiente a la } \\
\text { información }\end{array}$ & $\begin{array}{l}\text { Diseña y pone en } \\
\text { práctica un plan } \\
\text { de búsqueda de } \\
\text { información que } \\
\text { necesita. }\end{array}$ & $\begin{array}{l}\text { Identifica fuentes } \\
\text { de información } \\
\text { relevantes para } \\
\text { realizar la } \\
\text { búsqueda. }\end{array}$ & $\begin{array}{l}\text { Identifica fuentes } \\
\text { variadas y de } \\
\text { diverso origen, } \\
\text { aunque no } \\
\text { selecciona las } \\
\text { idóneas. }\end{array}$ & $\begin{array}{l}\text { No identifica } \\
\text { fuentes y accede } \\
\text { a cualquier } \\
\text { información. }\end{array}$ \\
\hline \multirow{3}{*}{$\begin{array}{l}\text { Evaluar la } \\
\text { información } \\
\text { y sus fuentes }\end{array}$} & $\begin{array}{l}\text { Valora la } \\
\text { adecuación de la } \\
\text { información a la } \\
\text { temática del } \\
\text { trabajo. }\end{array}$ & $\begin{array}{l}\text { Pone en relación } \\
\text { cada documento } \\
\text { seleccionado con } \\
\text { su necesidad de } \\
\text { información. }\end{array}$ & $\begin{array}{l}\text { Recoge información } \\
\text { acorde a la temática } \\
\text { de la actividad, sin } \\
\text { precisar su } \\
\text { pertinencia. }\end{array}$ & $\begin{array}{l}\text { Recoge cualquier } \\
\text { información } \\
\text { aunque no sea } \\
\text { adecuada para la } \\
\text { actividad. }\end{array}$ \\
\hline & $\begin{array}{l}\text { Valora la calidad } \\
\text { de la información. }\end{array}$ & $\begin{array}{l}\text { Selecciona los } \\
\text { recursos en } \\
\text { función de su } \\
\text { valor. }\end{array}$ & $\begin{array}{l}\text { Selecciona los } \\
\text { recursos, pero no } \\
\text { evalúa siempre } \\
\text { su calidad. }\end{array}$ & $\begin{array}{l}\text { No aplica criterios } \\
\text { para la selección } \\
\text { de recursos. }\end{array}$ \\
\hline & $\begin{array}{l}\text { Evalúa resultados } \\
\text { y se replantea la } \\
\text { búsqueda de } \\
\text { información. }\end{array}$ & $\begin{array}{l}\text { Identifica lagunas } \\
\text { y amplía las } \\
\text { fuentes } \\
\text { consultadas para } \\
\text { satisfacer la } \\
\text { necesidad de } \\
\text { información. }\end{array}$ & $\begin{array}{l}\text { Identifica lagunas } \\
\text { de información, } \\
\text { no siempre } \\
\text { recoge las fuentes } \\
\text { y su ubicación o } \\
\text { no sabe como } \\
\text { buscar lo que } \\
\text { necesita. }\end{array}$ & $\begin{array}{l}\text { Recopila la } \\
\text { información y sus } \\
\text { fuentes } \\
\text { deficientemente, } \\
\text { lo que le lleva a } \\
\text { ampliar las } \\
\text { búsquedas de } \\
\text { forma aleatoria } \\
\text { sin planificar. }\end{array}$ \\
\hline \multirow[t]{2}{*}{$\begin{array}{l}\text { Usar } \\
\text { eficazmente } \\
\text { la } \\
\text { información }\end{array}$} & $\begin{array}{l}\text { Organiza } \\
\text { internamente el } \\
\text { contenido. }\end{array}$ & $\begin{array}{l}\text { Utiliza una } \\
\text { secuencia lógica } \\
\text { de ideas y } \\
\text { plantamientos. }\end{array}$ & $\begin{array}{l}\text { Comete algunos } \\
\text { fallos al organizar } \\
\text { la secuencia } \\
\text { lógica de ideas. }\end{array}$ & $\begin{array}{l}\text { Va y vuelve sobre } \\
\text { las mismas ideas o } \\
\text { "da saltos en el } \\
\text { vacío". }\end{array}$ \\
\hline & $\begin{array}{l}\text { Expresa } \\
\text { sintéticamente el } \\
\text { contenido de la } \\
\text { información } \\
\text { consultada. }\end{array}$ & $\begin{array}{l}\text { Recoge las ideas } \\
\text { principales } \\
\text { utilizando sus } \\
\text { propias palabras. }\end{array}$ & $\begin{array}{l}\text { Recoge ideas } \\
\text { relevantes, } \\
\text { usando o no sus } \\
\text { propias palabras. }\end{array}$ & $\begin{array}{l}\text { Recoge y reúne } \\
\text { párrafos de diversa } \\
\text { procedencia sin } \\
\text { procesar la } \\
\text { información. }\end{array}$ \\
\hline \multirow[t]{2}{*}{$\begin{array}{l}\text { Usar } \\
\text { éticamente } \\
\text { las fuentes } \\
\text { de } \\
\text { información }\end{array}$} & $\begin{array}{l}\text { Demuestra } \\
\text { comprensión de } \\
\text { lo que constituye } \\
\text { plagio y no } \\
\text { presenta como } \\
\text { propios materiales } \\
\text { atribuibles a } \\
\text { autores. }\end{array}$ & $\begin{array}{l}\text { Fundamenta su } \\
\text { discurso escrito a } \\
\text { partir de las } \\
\text { fuentes consultadas. }\end{array}$ & $\begin{array}{l}\text { Incluye algunas } \\
\text { citas de los autores } \\
\text { consultados, pero } \\
\text { utiliza ideas sin } \\
\text { justificar. }\end{array}$ & $\begin{array}{l}\text { Transcribe párrafos } \\
\text { sin alusión a las } \\
\text { fuentes } \\
\text { consultadas. }\end{array}$ \\
\hline & $\begin{array}{l}\text { Selecciona un estilo } \\
\text { de referencias } \\
\text { apropiado y lo } \\
\text { utiliza de forma } \\
\text { consistente para } \\
\text { citar las fuentes. }\end{array}$ & $\begin{array}{l}\text { Aplica un estilo de } \\
\text { referencias } \\
\text { bibliográficas } \\
\text { normalizado. }\end{array}$ & $\begin{array}{l}\text { No utiliza un estilo } \\
\text { normalizado o } \\
\text { faltan algunos de } \\
\text { los datos que } \\
\text { permiten identificar } \\
\text { el documento. }\end{array}$ & $\begin{array}{l}\text { no suele recoger } \\
\text { las referencias } \\
\text { utilizadas o presenta } \\
\text { una lista de } \\
\text { referencias no } \\
\text { consultadas. }\end{array}$ \\
\hline
\end{tabular}

Fuente: Elaboración propia. 


\section{ResUltADOS Y DISCUSIÓN}

En cuanto a las habilidades relacionadas con el acceso, selección y evaluación de la información, se recogieron datos dirigidos a conocer su desarrollo inicial en el alumnado, así:

- En la dinámica grupal el alumnado identificó tres fuentes en las que tenía previsto comenzar la búsqueda de información: Wikipedia, Google y Dialnet. Sólo un estudiante hizo referencia a "Google Scholar», "Google Books», Redined, y la Biblioteca TNT (www.biblioteca.cchs.csic.es).

- Durante el proceso de búsqueda se observó que algunos estudiantes no buscaban correctamente y que no recogían adecuadamente aquellos datos que les permitiría la localización posterior de la información seleccionada, por lo que fue necesario hacerles preguntas dirigidas a que tomasen conciencia de la necesidad de completar el registro de la información seleccionada.

- La información contenida en la Tabla 1 mostró que:

- Todos los estudiantes realizaron sus búsquedas en la Wikipedia, en Google y en Dialnet. La información encontrada en la Wikipedia fue considerada válida para la actividad por el $88 \%$ de los grupos. En Google seleccionaron una media de 4,2 documentos, de los cuales evaluaron como válidos el 90,4\%. Mientras que en Dialnet encontraron una media de dos documentos por grupo, que evaluaron positivamente.

- Sólo el 44,4\% de los grupos utilizó Redined, seleccionando una media de un único documento que evaluaron de forma positiva.

- El 55,5\% de los estudiantes acudió a Google Books, seleccionando una media de 1,4 documentos por grupo. De estos documentos, fueron valorados positivamente el $71,4 \%$.

- Buscó información en Google Scholar el 44,4\%, seleccionando una media de dos documentos por grupo y considerando válidos un $62,5 \%$.

- A la Biblioteca TNT accedió un grupo, que seleccionó un documento que evaluó positivamente.

En un segundo momento, tras la explicación de distintas bases de datos y procedimientos de búsqueda, se recogió la Tabla 1 con la intención de conocer la evolución en las habilidades del alumnado. Estos datos permitieron dibujar el perfil de desarrollo de las habilidades relacionadas con el acceso y evaluación de la información (Tabla 3), teniendo en cuenta los indicadores de la Tabla 2:

- Todos los estudiantes identificaron fuentes variadas, aunque no siempre las utilizaron adecuadamente, detectándose dificultades en la elaboración de la ecuación de búsqueda. Algunos seleccionaron un único documento que utilizaron para elaborar la actividad, sin contrastar la información, quizá producto de la comodidad o bien porque les pareció fiable.

- Todos los estudiantes pusieron en relación cada documento seleccionado con su necesidad de información y mostraron un uso diferencial de las fuentes en función de la tarea. 
- El 83,3\% tuvo en cuenta diversos criterios de calidad a la hora de valorar recursos procedentes de Internet frente a un 16,7\% que mostró dificultades al conceder poca importancia a la autoría y fecha de creación.

- El 83,3\% de los estudiantes identificó algunas lagunas de información respecto a la búsqueda realizada. En este proceso encontraron bastantes dificultades puesto que unos grupos no recogieron la información necesaria para acceder a los documentos seleccionados, mientras que otros grupos, al no conceder el valor adecuado a las fuentes y documentos, no detectaron todas las lagunas y realizaron una ampliación sesgada. Tras ampliar la búsqueda, el 16,7\% obtuvo nuevos documentos más apropiados a la temática abordada.

Tabla 3. Niveles respecto al acceso y evaluación de la información.

\begin{tabular}{|l|c|c|c|}
\hline \multirow{2}{*}{ Dimensiones } & \multicolumn{2}{|c|}{ Niveles } \\
\cline { 2 - 4 } & Nivel I & Nivel II & Nivel III \\
\hline $\begin{array}{l}\text { Identifica fuentes de información relevantes para } \\
\text { realizar la búsqueda. }\end{array}$ & $0 \%$ & $100 \%$ & $0 \%$ \\
\hline $\begin{array}{l}\text { Pone en relación cada documento seleccionado } \\
\text { con su necesidad de información. }\end{array}$ & $100 \%$ & $0 \%$ & $0 \%$ \\
\hline Selecciona los recursos en función de su valor. & $83,3 \%$ & $16,7 \%$ & $0 \%$ \\
\hline $\begin{array}{l}\text { Identifica lagunas y amplía las fuentes consultadas } \\
\text { para satisfacer la necesidad de información }\end{array}$ & $16,7 \%$ & $83,3 \%$ & $0 \%$ \\
\hline
\end{tabular}

Fuente: Elaboración propia.

En cuanto a las habilidades relacionadas con el uso eficaz y ético de las fuentes de información para realizar la actividad, el documento recogido a cada uno de los estudiantes en la Fase II (valorado a partir de los indicadores de logro de la Tabla 2), junto a las observaciones desarrolladas en el aula, permitieron dibujar el perfil de desarrollo de estas habilidades. Los datos obtenidos, recogidos sintéticamente en la Tabla 4, muestran que:

- En cuanto a la organización de ideas y su secuencia, los estudiantes se dividieron al 33,3\% entre aquellos que: 1) utilizaron una secuencia lógica de ideas y planteamientos; 2) hicieron una organización de las ideas no siempre coherente; 3) no organizaron las ideas, sino que iban y venían sobre ellas, presentando avances y retrocesos.

- El 44,5\% de los estudiantes recogió y reunió párrafos de diversa procedencia sin procesar la información, copiando y pegando éstos de forma consecutiva para construir su propio texto. El 22,2\% del alumnado redactó el texto a partir de lo que había leído, auque no siempre fue relevante lo que recogió, dado que no profundizó lo suficiente en la temática a trabajar. Además, la redacción era muy simple, utilizando un exceso de frases cortas, no uniendo las ideas, lo que 
les Ilevaba a emplear demasiado el punto y aparte y, en ocasiones, cambiando únicamente algunas palabras a un párrafo que habían leído en alguno de los documentos consultados, para seguidamente introducirlo en el texto. Únicamente el 33,3\% recogió las ideas principales de los textos leídos utilizando sus propias palabras.

- El 88,9\% de los estudiantes presentó como propios los materiales de diversos autores, transcribiendo párrafos sin alusión a las fuentes consultadas. Sólo el $11,1 \%$ incluyó la referencia a algunos autores cuando utilizó sus ideas, aunque no lo hicieron en todas las ocasiones o les faltaban datos identificativos como el año o la página.

- El 22,2\% de los estudiantes recogió el material que utilizó para la realización de la actividad en una bibliografía, aunque no aplicó un estilo bibliográfico. Por ejemplo, cuando se trataba de información de Internet únicamente señalaban la URL, olvidando datos tan importantes como la autoría, la fecha de publicación, etc. Además, los estudiantes que señalaron alguna fuente fue porque preguntaron al profesorado si tenían que hacerlo, por lo que el $77,8 \%$ no elaboró una bibliografía.

Tabla 4. Niveles iniciales respecto a uso eficaz y ético de la información.

\begin{tabular}{|l|c|c|c|c|c|c|}
\hline \multirow{2}{*}{\multicolumn{1}{|c|}{ Dimensiones }} & \multicolumn{5}{c|}{ Niveles } \\
\cline { 2 - 7 } & \multicolumn{2}{|c|}{ Nivel I } & \multicolumn{2}{c|}{ Nivel II } & \multicolumn{2}{c|}{ Nivel III } \\
\cline { 2 - 7 } & I & F & I & F & I & F \\
\hline $\begin{array}{l}\text { Utiliza una secuencia } \\
\text { lógica de ideas y } \\
\text { planteamientos. }\end{array}$ & $33,33 \%$ & $66,66 \%$ & $33,33 \%$ & $33,33 \%$ & $33,33 \%$ & $0 \%$ \\
\hline $\begin{array}{l}\text { Recoge las ideas } \\
\text { principales utilizando } \\
\text { sus propias palabras. }\end{array}$ & $33,3 \%$ & $66,66 \%$ & $22,2 \%$ & $33,3 \%$ & $44,5 \%$ & $0 \%$ \\
\hline $\begin{array}{l}\text { Fundamenta su } \\
\text { discurso escrito a } \\
\text { partir de las fuentes } \\
\text { consultadas. }\end{array}$ & $0 \%$ & $50 \%$ & $11,1 \%$ & $50 \%$ & $88,9 \%$ & $0 \%$ \\
\hline $\begin{array}{l}\text { Aplica un estilo de } \\
\text { referencias } \\
\text { bibliográficas } \\
\text { normalizado. }\end{array}$ & $0 \%$ & $66,66 \%$ & $22,2 \%$ & $33,3 \%$ & $77,8 \%$ & $0 \%$ \\
\hline
\end{tabular}

Fuente: Elaboración propia. 
Una vez explicadas las cuestiones relacionadas con el uso eficaz y ético de las fuentes de información y aclaradas las dificultades encontradas por cada grupo en la tutoría, éstos elaboraron una versión definitiva de la «Wiki», con acceso directo desde el sitio Web de cada grupo y que puede ser consultada en Internet (Figura 1 y Figura 2). En esta última versión se observa una evolución del alumnado en algunas de las habilidades anteriormente mencionadas, como son: 1) Se duplicó tanto el número de grupos que utilizaron tanto una secuencia lógica en la exposición de ideas y planteamientos como el de los que se expresaron con sus propias palabras, llegando al 66,66\%. 2) El 50\% de los grupos fundamentó su discurso a partir de las fuentes consultadas. 3) El 66,66\% aplicó un estilo de referencias bibliográficas.

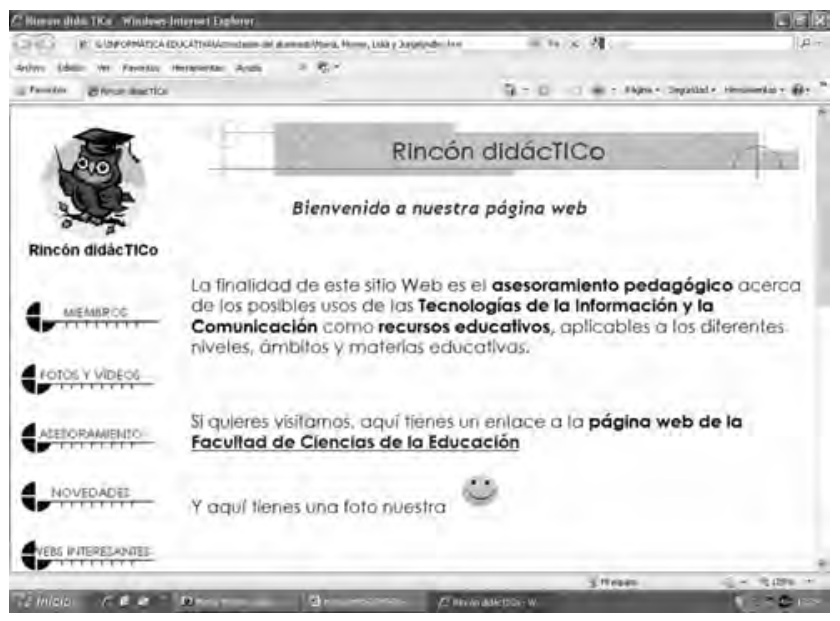

Figura 1. Imagen sitio Web de un grupo.

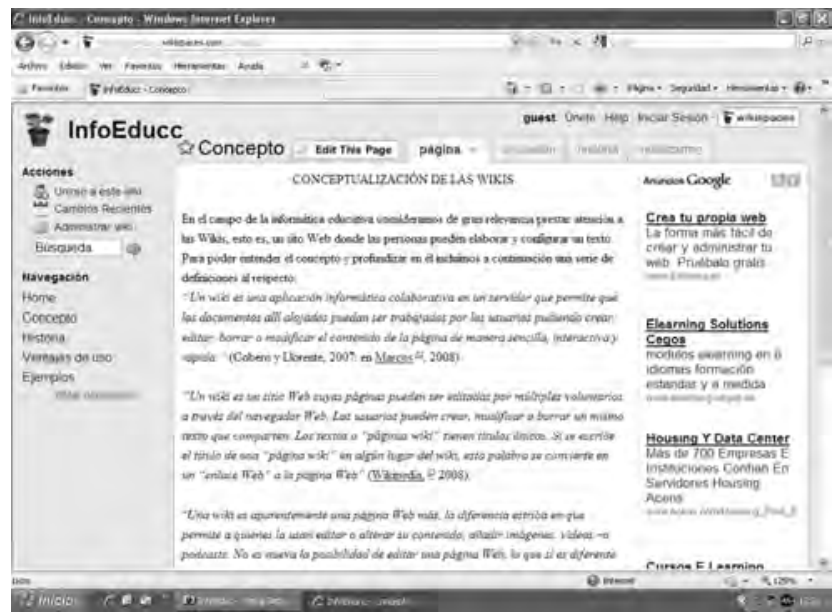

Figura 2. Imagen de una wiki grupal. 


\section{Conclusiones}

Se puede afirmar que esta experiencia fue positiva para el alumnado, dado que avanzó en el desarrollo de algunas habilidades implicadas en la gestión de la información, como muestran los resultados comentados anteriormente. Partir de los conocimientos previos manifestados por el alumnado para hacerle avanzar hacia un nivel de logro superior de las habilidades trabajadas, fue un aspecto positivo. No obstante, inicialmente los estudiantes mostraron un nivel inferior al que sería deseable en un universitario, dado que no tenían interiorizados métodos de trabajo que incluyesen la puesta en marcha de las habilidades relacionadas con esta competencia. Por ello, sería aconsejable introducir esta competencia en los objetivos y métodos de trabajo de las diferentes asignaturas. Su desarrollo no debería depender únicamente de la voluntad del profesor sino que debería ser el resultado del trabajo conjunto y secuencial de todos los docentes de cada titulación. Sólo así, los estudiantes podrán interiorizar unos procedimientos de trabajo adecuados a la sociedad en la que viven, rica en información, y aplicarlos habitualmente tanto en su vida académica como cotidiana. En este sentido, Gómez y Benito (2001) afirman que el profesor debe incluir entre las prácticas de la asignatura actividades documentales, supervisándolas, corrigiéndolas y valorándolas, revisando así el proceso de aprender a informarse del estudiante.

Ciertamente, el desarrollo de la competencia en gestión de la información no debe depender únicamente de la voluntad del profesor, sino que debe ser el resultado del trabajo conjunto de todos los docentes de cada titulación, siendo importante que esta formación esté integrada dentro del sistema nacional de enseñanza (United Nations Educational, Scientific and Cultural Organization, 2008, anexo, p.10). En esta línea, es preciso prever una formación escalonada a lo largo de toda la enseñanza universitaria, en espiral con refuerzos sucesivos. Ésta debe comenzar con una formación inicial obligatoria para asegurar que el conjunto de los estudiantes sea capaz de localizar, acceder, seleccionar y valorar la información y sus fuentes en su campo de conocimiento.

Además, para el éxito formativo en esta competencia sería beneficioso articular la formación universitaria con la desarrollada en las diferentes etapas del sistema educativo, pues se trata de aptitudes y métodos de trabajo que hay que hacer evolucionar desde la infancia a lo largo del tiempo (Comité de las Regiones, 2006). Se trata, pues, de integrarla en el sistema nacional de enseñanza, tal y como se está haciendo en España en el currículo de Educación Primaria (Ministerio de Educación y Ciencia, 2007a: art.6) y de Educación Secundaria Obligatoria (ESO) (Ministerio de Educación y Ciencia, 2007b: art. 6), bajo la denominación de "tratamiento de la información y competencia digital".

\section{REFERENCIAS BIBLIOGRÁFICAS}

AGENCIA NACIONAL DE EVALUACIÓN, CALIDAD Y ACREDITACIÓN (2005). Libro Blanco. Título de Grado en Pedagogía y Educación Social. Volumen 1. Madrid: ANECA. Recuperado el 24 de enero de 2008, de http://www.aneca.es/ activin/docs/libroblanco_pedagogia1_0305.pdf. 
AMERICAN LIBRARY ASSOCIATION (1989). Final Report. Chicago: ALA. Recuperado el 25 de marzo de 2009, de http://www.ala.org/ala/mgrps/divs/acrl/publications/ whitepapers/presidential.cfm.

AMERICAN LIBRARY ASSOCIATION (2000). Information Literacy Competency Standards for Higher Education. Chicago: ACRL. Recuperado el 28 de octubre de 2008, de http://www.ala.org/ala/mgrps/divs/acrl/standards/standards.pdf.

ASSOCIATION OF COLLAGE AND RESEARCH LIBRARIES (2008). Political Science Research Competency Guidelines. Recuperado el 13 de mayo de 2009, de http://www.ala.org/ala/mgrps/divs/acrl/standards/PoliSciGuide.pdf.

AUSTRALIAN LIBRARY AND INFORMATION ASSOCIATION (2003). A library advocate's guide to building information literate communities. ALIA. Recuperado el 11 de diciembre de 2008, de http://alia.org.au/advocacy/literacy.kit.pdf.

AUSTRALIAN AND NEW ZEALAND INSTITUTE FOR INFORMATION LITERACY (2003). El marco para la alfabetización informacional en Australia y Nueva Zelanda. Principios, normas y práctica. Boletín de la Asociación Andaluza de Bibliotecarios, 73, 109-120. Recuperado el 8 de octubre de 2007, de http:// www.aab.es/pdfs/baab73/73a4.pdf.

BERNHARD, P. (2002). La formación en el uso de la información: una ventaja en la enseñanza superior. Situación actual. Anales de Documentación, 5, 409-435.

BRITISH SOCIETY OF COLLEGE, NATIONAL AND UNIVERSITY LIBRARIES (2003). Information skills in higher education: a SCONUL position paper. Recuperado el 19 de mayo de 2006, de http://www.sconul.ac.uk/groups/information_literacy/ papers/Seven_pillars.html.

CALDERÓN, A. y DE JORGE, M. (2006). Competencias en información y entorno tecnológico. Recuperado el 5 de febrero de 2009, de http://observatorio.cnice. mec.es/modules.php?op=modload $\&$ name $=$ News\&file $=$ article $\&$ sid $=335$.

COMITÉ DE LAS REGIONES (2006, 22 de septiembre). Dictamen del Comité de las Regiones sobre la Propuesta de recomendación del Parlamento Europeo y del Consejo sobre las competencias clave para el aprendizaje permanente. Diario Oficial de las Comunidades Europeas, C 229, 21-28.

COUNCIL OF AUSTRALIAN UNIVERSITY LIBRARIANS (2002). Normas sobre alfabetización en información, 2001 (1aㅡ Edición). Boletín de la Asociación Andaluza de Bibliotecarios, 68, 67-90.

COUNCIL OF AUSTRALIAN UNIVERSITY LIBRARIANS (2004). El marco para la alfabetización informacional en Australia y Nueva Zelanda. Principios, normas y práctica. (2a Edición). Recuperado el 21 de octubre de 2008, de http://www.aab. es/pdfs/gtbunormas08.pdf.

GÓMEZ, J. A. y BENITO, F. (2001). De la formación de usuarios a la alfabetización informacional. Propuestas para enseñar las habilidades de información. Scire, 7 (1), 53-83.

MINISTERIO DE EDUCACIÓN Y CIENCIA (2007a, 20 de julio). Orden ECI/2211/ 2007, de 12 de julio, por la que se establece el currículo y se regula la ordenación de la Educación Primaria. Boletín Oficial del Estado, 173, 31487-31566. 
MINISTERIO DE EDUCACIÓN Y CIENCIA (2007b, 21 de julio). Orden ECI/2220/ 2007, de 12 de julio, por la que se establece el currículo y se regula la ordenación de la Educación secundaria obligatoria. Boletín Oficial del Estado, 174, 31680-31828.

MINISTERIO DE EDUCACIÓN Y CIENCIA (2007c, 29 de diciembre). Orden ECI/ $3854 / 2007$, de 27 de diciembre, por la que se establecen los requisitos para la verificación de los títulos universitarios oficiales que habiliten para el ejercicio de la profesión de Maestro en Educación Infantil. Boletín Oficial del Estado, 312, 53735-53738.

MINISTERIO DE EDUCACIÓN Y CIENCIA (2007d, 29 de diciembre). Orden ECI/ $3857 / 2007$, de 27 de diciembre, por la que se establecen los requisitos para la verificación de los títulos universitarios oficiales que habiliten para el ejercicio de la profesión de Maestro en Educación Primaria. Boletín Oficial del Estado, 312, 53747-53750.

MOLINA, S., IGLESIAS, Ma T. y DIEGO, C. (2008). Formación en gestión de la información sobre educación con materiales multimedia. Píxel Bits. Revista de medios y educación, 31, 71-89.

PINTO, M., SALES, D. y MARTíNEZ-OSORIO, P. (2009). El personal de la biblioteca universitaria y la alfabetización informacional: de la autopercepción a las realidades y retos formativos. Revista Española de Documentación Científica, 32 (1), 60-80.

UNITED NATIONS EDUCATIONAL, SCIENTIFIC AND CULTURAL ORGANIZATION \& NATIONAL FORUM ON INFORMATION LITERACY (2003). Declaración de Praga. Recuperado el 11 de marzo de 2009, de http://www.melangeinfo. com/Doc/Declaraci_Praga_castellano.pdf.

UNITED NATIONS EDUCATIONAL, SCIENTIFIC AND CULTURAL ORGANIZATION \& NATIONAL FORUM ON INFORMATION LITERACY (2005). Proclamación de Alejandría acerca de la alfabetización informacional y el aprendizaje de por vida. Recuperado el 15 de mayo de 2009, de http://www.infolit.org/International_ Colloquium/alexprocspa.doc.

UNITED NATIONS EDUCATIONAL, SCIENTIFIC AND CULTURAL ORGANIZATION (2008, 4 agosto). Informe del director general sobre un proyecto de plan estratégico del Programa Información Para Todos (PIPT), revisado por el consejo intergubernamental del PIPT. 180 a Reunión del Consejo Ejecutivo de la UNESCO. Paris: UNESCO. Recuperado el 18 de junio de 2009, de http://unesdoc.unesco. org/images/0016/001618/161860s.pdf.

UNIVERSIDAD DE OVIEDO (2000, 30 agosto). Resolución de 8 de agosto de 2000, de la Universidad de Oviedo, por la que se publica la adaptación del plan de estudios de Licenciado en Pedagogía a los Reales Decretos 614/1997, de 25 de abril, y 779/1998, de 30 de abril. Boletín Oficial del Estado, 208, 30797-30810.

WEBBER, S. y JOHNSTON, B. (2006). Hacia una universidad alfabetizada en información. Boletín de la Asociación Andaluza de Bibliotecarios, 84-85, 47-52. 